\title{
Preparation and Holographic Recording of Fluorescent Photopolymer Films Containing Anthracene Polymer for Security
}

\author{
Teahoon Park, Yoonjung Kim, Jeonghun Kim, and Eunkyoung Kim* \\ Department of Chemical and Biomolecular Engineering, Yonsei University, \\ 262 Seongsanno, Seodaemun-gu, Seoul 120-749, Korea
}

(Received October 19, 2010 : revised November 23, 2010 : accepted November 23, 2010)

\begin{abstract}
Photopolymer films containing fluorescent anthracene polymer, polymethyleneanthracene (PMAn), were prepared with different concentrations of PMAn for holographic recording useful for security documents. The fluorescent photopolymer film showed enhanced fluorescent intensity due to the micro-separation which arose from grating formation and diffusion during photopolymerization. Experimental values of diffraction efficiency were well matched to the simulated values for photopolymers having different PMAn concentrations. Holography patterning was carried out using the fluorescent photopolymer under a photo-mask. A grating was confirmed using microscope techniques in the recorded area under the pattern. Importantly the recorded area showed enhanced fluorescence compared to the unrecorded part, allowing fluorescence patterns at micro scale along with the submicron grating pattern. The fluorescence pattern recorded on the photopolymer film provides additional readability of holographic reading and thus is useful for secure recording and reading of information.
\end{abstract}

Keywords : Holography, Photopolymer, Fluorescent pattern, Security film

OCIS codes : (090.0090) Holography; (210.0210) Optical data storage; (050.0050) Diffraction and gratings

\section{INTRODUCTION}

Photopolymer has been researched and developed for semi-conductor applications. It is usually used as coating materials in industrial fields. There are three types of photopolymers - liquid, liquid crystal, and dry film. Photopolymer consists of monomer, sensitizer, initiator, and binder. The monomer has a functional group such as acrylate or epoxy. The initiator has sensitivity for a specific wavelength, so the wavelength of the laser can be selected by changing initiator and sensitizer. The composition of photopolymer should be optimized to get high sensitivity and diffraction efficiency [1-8]. Recently, there have been a lot of efforts to save and store more information [9-10]. For this purpose, there has been much research to develop hologram storage materials and tools. The photopolymers are achieving the spotlight in the holographic field. They can reduce the cost of production. The synthesis of photopolymer is not too difficult and they are more cost effective than inorganic materials. Holograms are widely used in paper money, gift certificates, securities, and in many applications for holographic security media systems.

To satisfy these interests, many kinds of photopolymer which have different functional groups have been studied [14-19]. Holographic recording results from interference of the light in photopolymer film. During the recording, monomers diffused in the media and thus optical properties such as the refractive index of the photopolymer film are changed. By adding fluorescent polymer in the photopolymer film, the color and fluorescent intensity are changed. Using these properties, dual security systems can be possible [20]. Herein we report properties of photopolymer film containing fluorescent polymer and its applications for holographic recording and security film.

\section{EXPERIMENTAL}

\subsection{Materials}

Ethylene glycol phenyl ether acrylate (PA) and polysulfone

\footnotetext{
*Corresponding author: eunkim@yonsei.ac.kr

Color versions of one or more of the figures in this paper are available online.
} 
(PES, $\mathrm{Mw}=35,000)$ were purchased from Aldrich. The triazine dimethacrylate (DT) was synthesized from cyanuric chloride, according to the literature [11]. Bis $\left(\mu^{5}-2,4\right.$ cyclopentadien-1-yl)-bis-[2,6-difluoro-3-(1Hpyrrol-1-yl) phenyl] titanium (Irgacure 784) was purchased from Ciba Geigy and used as a photo-radical initiator. PMAn (polymethylanthracene, $\mathrm{Mw}=3650$ ) used as fluorescent polymer was synthesized using a reported procedure [21]. The solvent chloroform and 1, 1, 2, 2-tetrachloroethane (TCE) were purchased from Aldrich.

\subsection{Preparation of photopolymer films}

The photosensitive compositions consisted of a mixture of monomers (40 wt\%), polysulfone (60 $\mathrm{wt} \%)$, a photoinitiator and PMAn. PA is a monofunctional monomer and DT is a difunctional monomer. Polysulfone $(0.75 \mathrm{~g}, \mathrm{Mw}=$ $35,000)$ was dissolved in chloroform and TCE. The polysulfone solution was a mixture of $0.5 \mathrm{~g}$ of monomers, $0.013 \mathrm{~g}$ of Irgacure 784 and $2 \mathrm{wt} \%$ of PMAn in a brown bottle. Because of the Irgacure 784, the solution color is yellow. The yellow mixture was stirred at $150 \mathrm{rpm}$ for 3 $\mathrm{h}$ with a magnetic stirrer, and filtered using membranes of $0.45 \mu \mathrm{m}$ pore size attached to a Teflon syringe. The solution was coated on a slide glass by spin coating. The films were prepared with different PMAn concentrations of thickness $\sim 50 \mu \mathrm{m}$ and dried for $6 \mathrm{~h}$.

\subsection{Holographic set-up}

Holographic recording was generated by a series optical setup consisting of a diode laser $(491 \mathrm{~nm})$, polarizer, beam splitter, and detector, as reported before (Fig. 1) [14]. A power ratio of the object and reference beams was fixed as $1: 1$ by the adjustable polarized beam-splitter (PBS). The two beams were coherent on the photopolymer films and the interference pattern was generated at the films. The diffraction efficiency (DE) was determined from the ratio of the diffraction intensity (ID) against the sum of ID and intensity of transmission beam (IT). The blocking time of

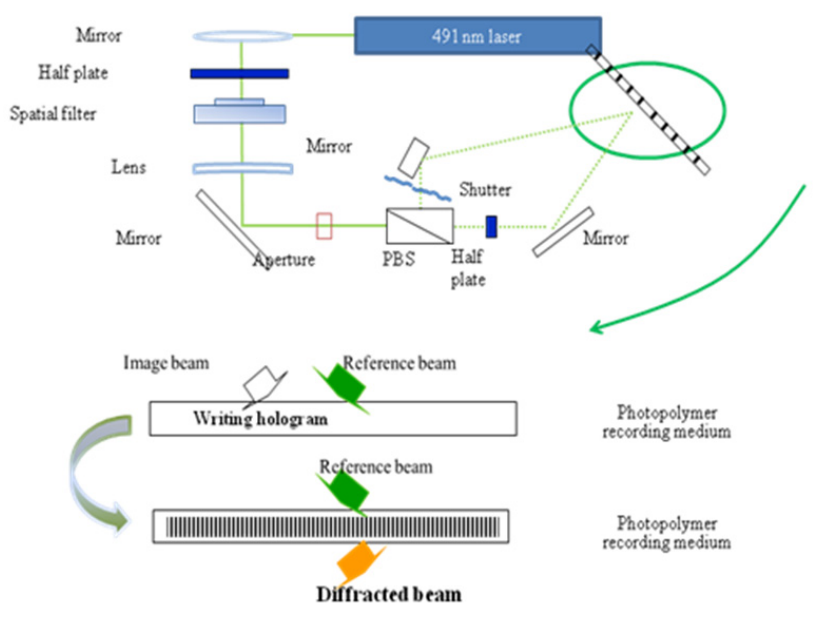

FIG. 1. Holographic recording set-up. the electrical shutters to measure diffraction efficiency of the photopolymer was programmed to be 0.5 second.

\section{RESULTS AND DISCUSSION}

\subsection{Diffraction efficiency of photopolymer film containing fluorescent material}

The yellow color photopolymer films which containing fluorescent polymer PMAn were prepared. Absorption spectra of this photopolymer film showed high absorption around $490 \mathrm{~nm}$ (Fig. 2a). This is due to Irgacure 784 absorption. Thus the absorption spectra were independent of PMAn concentration. The absorption at the $490 \mathrm{~nm}$ region makes holographic recording feasible on this photopolymer film at $491 \mathrm{~nm}$. PMAn was stable during holographic recording [15-16].

The diffraction efficiency (DE) growth for the photopolymer film containing different PMAn concentrations is shown in Fig. 2 (b). The photopolymer film without PMAn was employed as a reference which showed maximum $22 \%$ of DE. The photopolymer film containing $2 \mathrm{wt} \%$ PMAn showed the highest DE among the samples. As PMAn concentration was increased over $2 \mathrm{wt} \%$, DE decreased and the
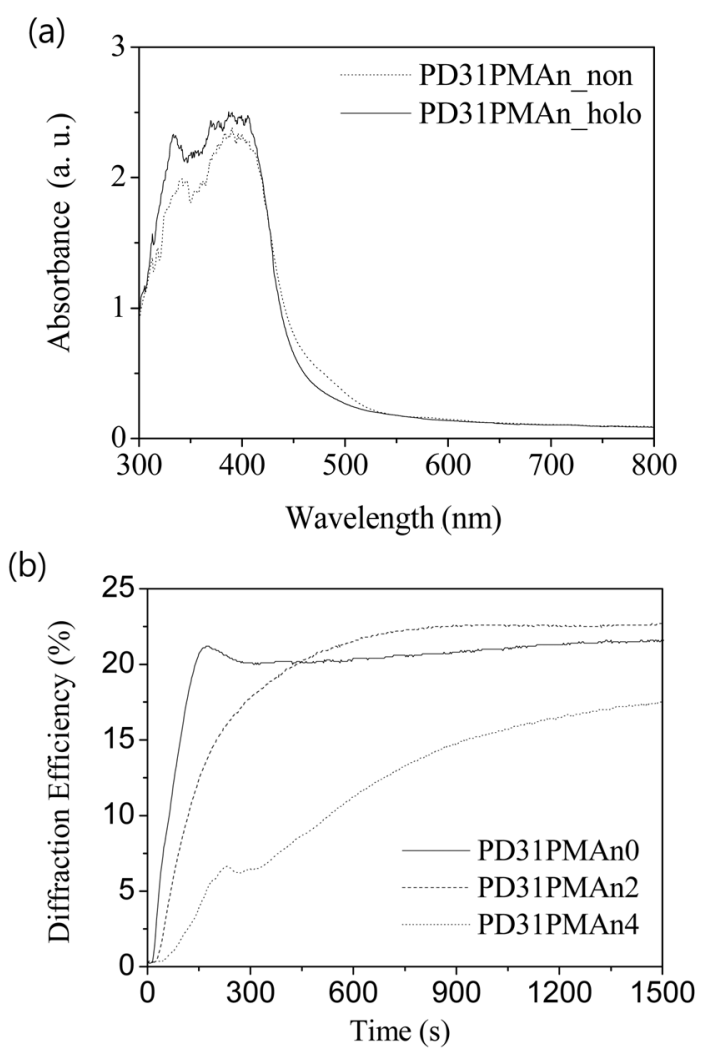

FIG. 2. (a) UV-Vis spectra of photopolymer film (holographic patterned part: solid line, non- pattern part: dotted line) and (b) diffraction efficiency against recording time for the photopolymer film containing different concentrations of PMAn. 
growth rate of DE was also reduced. These results could be ascribed to PMAn, which has a large enough size to disturb the diffusion as well as polymerization of monomers during the holographic recording.

\subsection{Polymerization time by photo DSC}

To examine the effect of PMAn on the polymerization of monomers in the photopolymer, we determined the polymerization time in the presence of PMAn by a photo DSC. The photopolymer solution was dropped on an aluminum substrate and dried [11]. Diffusion time was determined from the diffusion model according to the equation (Eq. (1)).

$$
\frac{\partial \phi^{(m)}}{\partial t}=-k_{R}(t) I^{\delta}(x) \phi^{(m)}(x, t)+\frac{\partial}{\partial x} D \frac{\partial}{\partial x} \phi^{(m)}(x, t)
$$

Where $\phi$ stands for the volume fractions of the different compounds; with $\phi^{(\mathrm{m})}$ and $\phi^{(\mathrm{p})}$ standing for monomer and polymer, respectively; $D$ is the diffusion constant; $I$ is the illumination intensity, $\delta$ is the reaction rate constant, and $k_{R}(t)$ is the polymerization rate. The polymerization rate values were measured by the photo DSC according to our previous reports [22]. Diffusion time was calculated by the simulation with Eq. (1) and Matlab software, as described below.

The polymerization and diffusion time were significantly longer when PMAn content was higher as summarized in Table 1 . This clearly verifies that PMAn disturbs the monomer's polymerization and diffusion, which are important for high diffraction efficiency. The optimized condition of monomer ratio between PA:DT to get high DE was found to be 3:1 (PD31).

\subsection{Simulation}

DE is calculated theoretically according to above Eq. (1) using the values of the polymerization time and diffusion time determined above in Table $1 . I$ and $\phi$ are experimental values, $k_{R}(t)$ was measured and calculated using Photo-DSC. With these values, other parameters could be obtained by simulation. As shown in Fig. 3, the growth of $\mathrm{DE}$ obtained from simulation was well matched with experiment results.

\subsection{Fluorescence intensity changes}

The fluorescence intensity after the holographic patterning

TABLE 1. Polymerization time and diffusion time of photopolymer films

\begin{tabular}{ccccc}
\hline \hline Sample & $\begin{array}{c}\text { Polymerization } \\
\text { time }(\mathrm{s})\end{array}$ & $\begin{array}{c}\text { Diffusion } \\
\text { time }(\mathrm{s})\end{array}$ & DE & $\begin{array}{c}\text { Thickness } \\
(\mu \mathrm{m})\end{array}$ \\
\hline PD31PMAn0 & 23 & 77 & 0.21 & 54 \\
PD31PMAn2 & 87 & 579 & 0.23 & 58 \\
PD31PMAn4 & 204 & 2065 & 0.19 & 53 \\
\hline
\end{tabular}

was enhanced as compared to the unpatterned sample. The fluorescent spectra of the photopolymer film before and after holographic recording are compared in Fig. 5. It showed clearly the enhancement of the fluorescence intensity in the broad emission region after patterning compared to the unpatterned sample. This may be ascribed to the micro separation of PMAn during the monomer diffusion and polymerization in the fluorescent polymer film [16]. This enhancement in FL allows further additional fluorescent patterning using a photo mask as described below. The fluorescence enhancement by holographic patterning was intact for 2 years as shown in Fig. 4 (b).

(a)

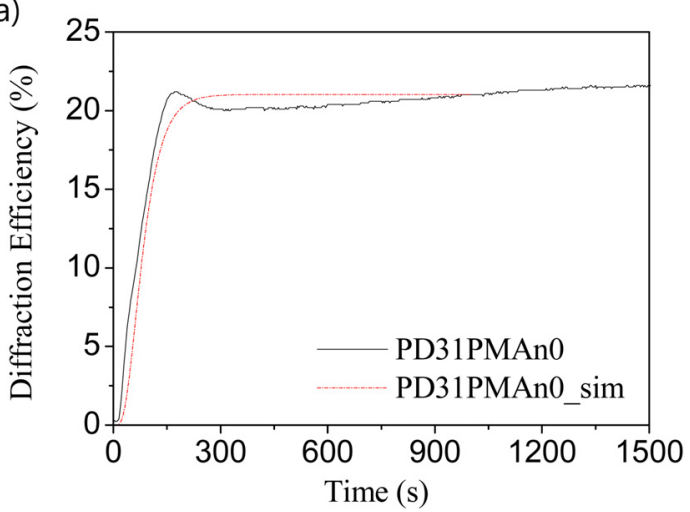

(b)

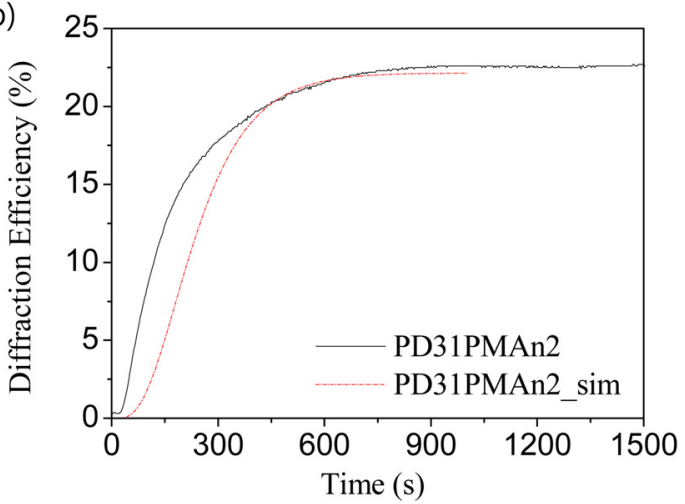

(c)

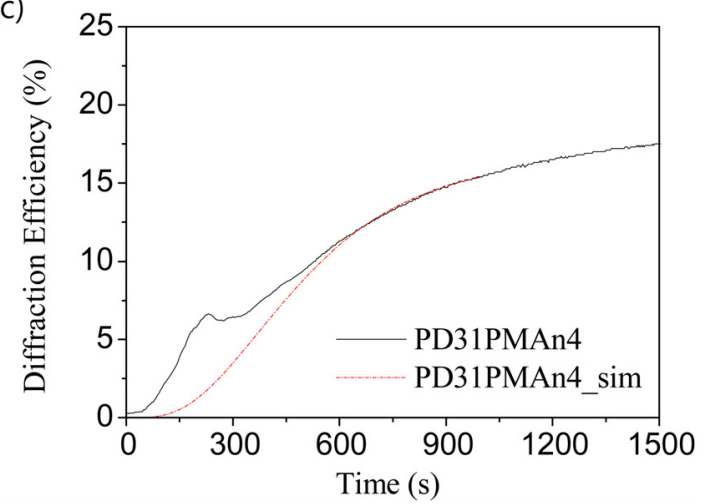

FIG. 3. Comparison of experimental diffraction efficiency with simulation values (experimental value: black solid line, simulated value: red dashed line) (a) PMAn 0 wt \%, (b) PMAn 2 wt \%, and (c) PMAn 4 wt \%. 
(a)
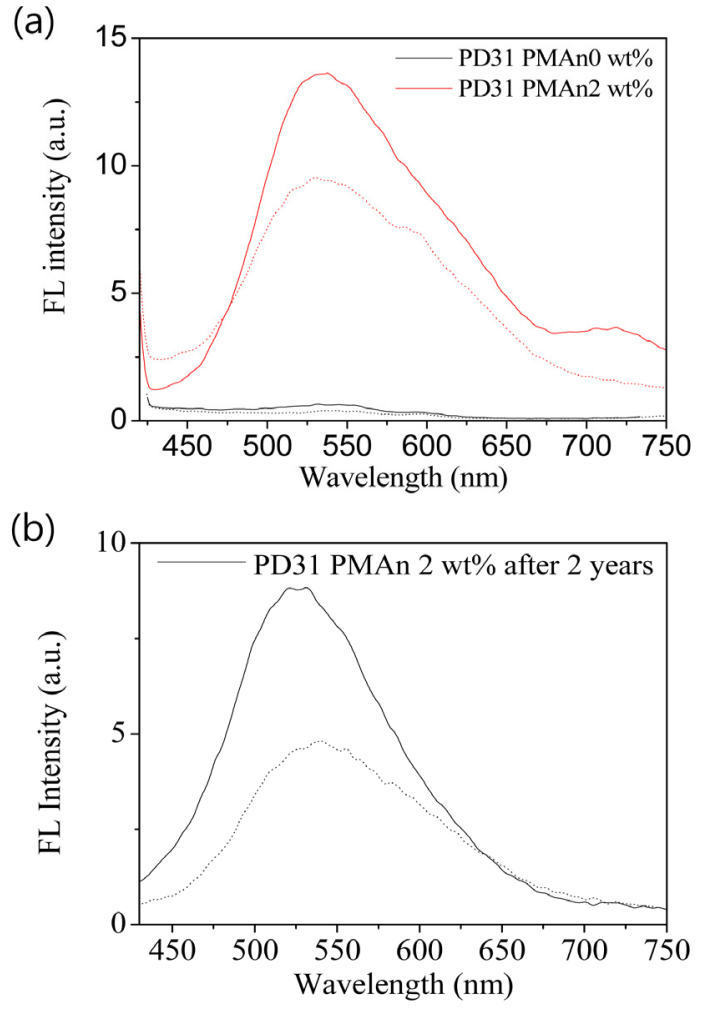

FIG. 4. Fluorescence (FL) intensity of the photopolymer containing PMAn 0 and 2 wt \% (holography unrecorded sample: dotted line, holography recorded sample: solid line) (a) 2 years before and (b) present.

\subsection{Secure fluorescent images}

Taking advantage of the enhancement in FL by holographic patterning, we attempted to inscribe a pattern using a photomask during the holographic patterning process. Fig. 5 shows the mask pattern image as well as grating image formed by holographic patterning. The mask pattern image was well defined under an optical microscope as shown in Fig. 5 (a) and (b). The uniform gratings formed by holographic patterning were clearly observed under an optical microscope as shown in Fig. 5 (c), showing a black line of $580 \mathrm{~nm}$ width for the polymerized region. The mask pattern image was well defined under a fluorescence microscope as shown in Fig. 5 (d). The fluorescent intensity was increased in the recorded part. Thus this holographic patterning with a photo mask affords double pattern formation. One is photo mask patterning and the other is grating pattern by holographic recording. From this double patterning, a dual secure system is achieved. One part is changes in fluorescent intensity by photo mask recording and the other is changes in refractive index pattern made by holographic recording. This holographic recording is a result of chemical reaction. It is stable for a long time. Fig. 6 shows a comparison of FL intensity and DE for the patterned image over 2 years, ensuring long term stability of the secure image.

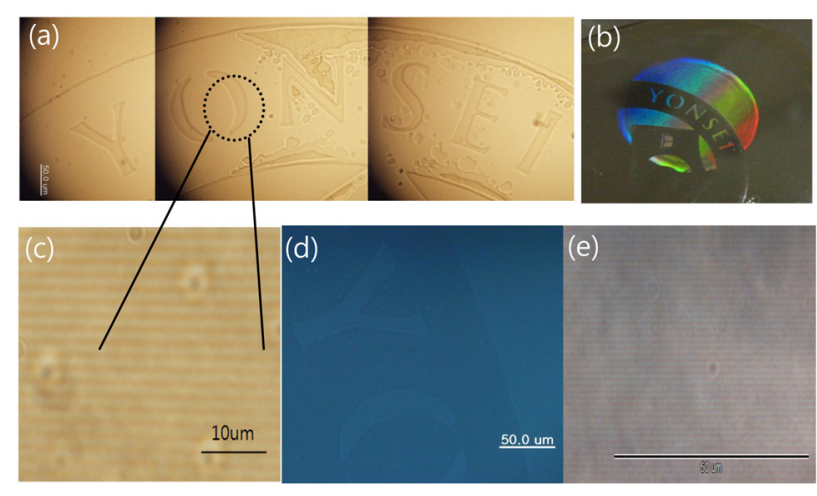

FIG. 5. Holographic pattern images taken 2 years before. (a) optical image, (b) sample image, (c) optical image of diffraction pattern, (d) fluorescent microscope image, and (e) present optical image of diffraction pattern.

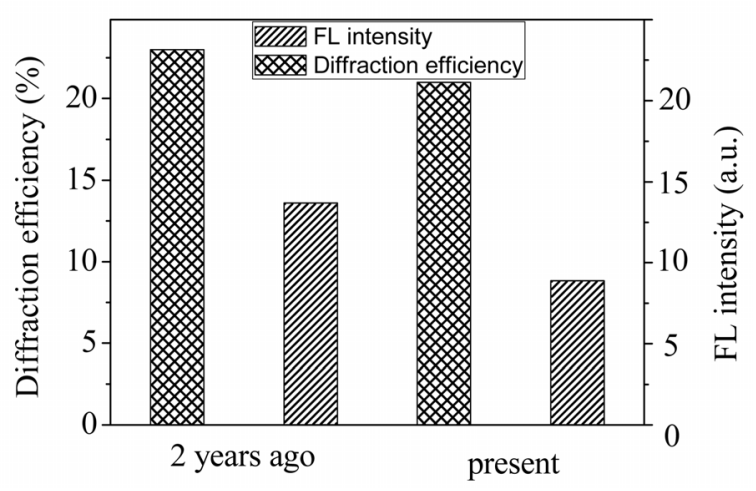

FIG. 6. Comparisons of FL intensity and DE for 2 years.

\section{CONCLUSION}

In this paper, the fluorescent photopolymer film containing PMAn was examined. The effects of PMAn on DE and fluorescent intensity of the photopolymer film were ascribed to the big size of fluorescent polymer, which disturbed the diffusion of monomers. Thus DE of the photopolymer containing PMAn was decreased when the concentration of PMAn was high. From photo DSC experiment, polymerization time and diffusion time were determined and it was found that the simulation result was matched to the experimental values. The enhancement of FL intensity of the photopolymer films after holographic patterning allowed double patterning with a fluorescent photopolymer film. Stable holographic pattern with a fluorescent pattern was formed by holographic recording under a photo mask. The pattern formation capability of the fluorescent photopolymer film containing PMAn can be exploited for secure films.

\section{ACKNOWLEDGMENT}

This research was supported by the Pioneer Research 
Center Program through the National Research Foundation of Korea funded by the Ministry of Education, Science and Technology (2010-0019313) and the National Research Foundation (NRF) grant funded by the Korea government (MEST) through the Active Polymer Center for Pattern Integration (No. R11-2007-050-00000-0).

\section{REFERENCES}

1. S. J. Woltman, J. N. Eakin, G. P. Crawford, and S. Žumer, "Holographic diffraction gratings using polymer-dispersed ferroelectric liquid crystals," Opt. Lett. 31, 3273-3275 (2006).

2. J. A. Arns, W. S. Colburn, and S. C. Barden, "Volume phase gratings for spectroscopy, ultrafast laser compressors, and wavelength division multiplexing," Proc. SPIE 3779, 313-323 (1999).

3. C. Wochnowski, Y. Cheng, K. Meteva, K. Sugioka, K. Midorikawa, and S. Metev, "Femtosecond-laser induced formation of grating structures in planar polymer substrates," J. Opt. A: Pure Appl. Opt. 7, 493-501 (2005).

4. Y. H. Cho, C. W. Shin, N. Kim, B. K. Kim, and Y. Kawakami, "High-performance transmission holographic gratings via different polymerization rates of dipentaerythritol acrylates and siloxane-containing epoxides," Chem. Mater. 17, 62636271 (2005).

5. R. A. Duarte-Quiroga, S. Calixto, and D. J. Lougnot, "Optical characterization and applications of a dual-cure photopolymerizable system," Appl. Opt. 42, 1417-1425 (2003).

6. E. Kim, J. Park, C. Shin, and N. Kim, "Effect of organic side-chains on the diffraction efficiency of an organic-inorganic hybrid nanocomposite film," Nanotechnology 17, 2899-2906 (2006)

7. H. Lee, B. Sarwade, E. Kim, and S. Lee, "Photopolymers containing triazine monomers for holographic recording," Proc. SPIE 6335, 63350R1-63350R6 (2006).

8. J. Joe, J. Lee, S. Yoon, S. Nam, and D. Kim, "Diffraction efficiency change in PVA/AA photopolymer films by $\mathrm{SeO}_{2}$ and $\mathrm{TiO}_{2}$ nano particle addition," Korean J. Opt. Photon. 21, 82-88 (2010).

9. J. S. Yi and Y. H. Lee, "Intermediate holographic data storage system by using sequentially superimposed recording," J. Opt. Soc. Korea 13, 456-463 (2009).
10. M.-S. Kim, G. Baasantseren, N. Kim, and J.-H. Park, "Hologram generation of 3D objects using multiple orthographic view images," J. Opt. Soc. Korea 12, 269-274 (2008).

11. K. Her, H. Jang, and D. Kim, "Diffraction efficiency improvement of $\mathrm{PVA} / \mathrm{AA} / \mathrm{SeO}_{2}$ photopolymer with various film thickness and eosin Y contents," Korean J. Opt. Photon. 20, 230-235 (2009).

12. K. Her and D. Kim, "Study of diffraction efficiency values for photopolymer films added $\mathrm{TiO}_{2}$ nanoparticles," Korean J. Opt. Photon. 20, 123-127 (2009).

13. H. Jung, N. Kim, J. Yoon, and T. Park, "Optical characteristic and image recording of reflection type photopolymer in transmission structure," Korean J. Opt. Photon. 18, 8-13 (2007).

14. H.-J. Lee, B. D. Sarwade, J. Park, and E. Kim, "Synthesis of new photopolymeric methacrylate thioester with s-triazine ring for holographic recording," Opt. Mater. 30, 637-644 (2007).

15. H. Oh, J. Kim, K. Rameshbabu, J. Do, and E. Kim, "Fluorescent grating patterns of photopolymer film containing ethylene glycol phenyl ether acrylate," Journal of Nanoscience and Nanotechnology 8, 4616-4620 (2008).

16. H. Oh, J. Kim, and E. Kim, "Holographic recording on photopolymers containing pyrene for enhanced fluorescence intensity," Macromolecules 41, 7160-7165 (2008).

17. J. Kim, H. Oh, and E. Kim, "A dual-functional monomer having epoxy and methacrylate group for holographic recording," Journal of Materials Chemistry 18, 4762-4768 (2008).

18. J. Kim, H. Oh, J. Yoo, K. Rameshbabu, and E. Kim, "Fluorescent photopolymer holographic patterning," Mol. Cryst. Liq. Cryst. 491, 67-73 (2008).

19. J. Kim and E. Kim, "Holographic security media prepared from photochromic fluorescent films," Proc. SPIE 7118, 71180F1-71180F10 (2008).

20. E. Kim, J. Park, S. Cho, N. Kim, and J. Kim, "Preparation and holographic recording of diarylethene-doped photochromic films," ETRI Journal 25, 253-257 (2003).

21. K. Rameshbabu, Y. Kim, T. Kwon, J. Yoo, and E. Kim, "Facile one-pot synthesis of a photo patternable anthracene polymer," Tetrahedron Lett. 48, 4755-4760 (2007).

22. H. Lee, H. Oh, E. Kim, D. Dung, and N. Kim, "Diffusion model of monomers in a photopolymer film for holographic recording," Proc. SPIE 6335, 63350M1-63350M8 (2006). 